\title{
THE MANIFOLD SMOOTHING PROBLEM ${ }^{1}$
}

BY STEWART S. CAIRNS

Communicated by A. M. Gleason, January 11, 1961

The Schoenflies Theorem in $n$ dimensions has been proved by both Marston Morse [4] and Morton Brown [1] subject to the shell hypothesis [4]. Morse's proof leads to $C^{m}$-diffeomorphisms. We now prove the following Schoenflies Theorem for polyhedra without the shell hypothesis.

THEOREM $1 .{ }^{2}$ Let $P^{n-1}$ be a combinatorial $(n-1)$-sphere in a euclidean $n$-space $E^{n}$, and let $N$ be an arbitrary neighborhood of $P^{n-1}$. Then $E^{n}$ can be mapped onto itself by a homeomorphism $h$ which is a $C^{\infty}$-diffeomorphism on $E^{n}-N$ and which maps $P^{n-1}$ onto a euclidean $(n-1)$-sphere $S^{n-1}$.

The proof commences with a modification of a procedure due to H. Noguchi [5] yielding an $\epsilon$-isotopy of $E^{n}$ carrying $P^{n-1}$, on $D^{n}$, into a polyhedron $Q^{n-1}$, admitting a transverse vector field. A neighborhood of $Q^{n-1}$ is fibred by $C^{\infty}-(n-1)$-spheres, which permits a completion of the proof with the aid of Morse's methods [4]. His exceptional interior point can be relegated to $N$. The proof is inductive, requiring a partial assumption of Theorem 1 in the next lower dimension.

Corollary. Given $a \delta>0, E^{n}$ admits a $\delta$-isotopy $h_{t}(0 \leqq t \leqq 1)$ such that (1) $h_{t}$ is the identity on the unbounded component of $E^{n}-N$, (2) $h_{t}\left(P^{n-1}\right) \subset D^{n}(t>0)$ and $(3) h_{t}\left(P^{n-1}\right)$ is a $C^{\infty}-(n-1)$-sphere $(t>\delta)$.

We will call a combinatorial $n$-manifold smoothable or nonsmoothable according as it is or is not compatible with a differentiable structure. The known nonsmoothable manifolds include a $K^{8}$ due to Milnor [3] and a $K^{10}$ due to Kervaire [2]. The latter is strongly nonsmooth$a b l e$, in the sense that the topological manifold it covers, $M^{10}=\left|K^{10}\right|$, can not carry a differentiable structure, either compatible or incompatible with $K^{10}$.

A piecewise differentiable imbedding of a $K^{m}$ in a differentiable $n$-manifold $M^{n}$ means a homeomorphism $h: K^{m} \rightarrow M^{n}$, where $h$ is differentiable of maximal rank on each closed simplex of $K^{m}$.

1 This work was supported by National Science Foundation Grant No. G14431.

2 A sharpening of this theorem appears in Proc. Nat. Acad. Sci. U.S.A. vol. 47, (1961) pp. 328-330. 
TheOREM 2. A combinatorial $n$-manifold $K^{n}$ without boundary is smoothable if and only if $K^{n}$ admits a piecewise differentiable imbedding $h$ into a differentiable $M^{n+1}$.

The necessity of the condition is easy to prove. The sufficiency proof commences with an $h: K^{n} \rightarrow M^{n+1}$ restricted, as in the proof of Theorem 1 , so that $h\left(K^{n}\right)$ admits a transverse vector field on $M^{n+1}$. Let $M^{n+1}$ be represented as a differentiable submanifold of an $E^{n+r}$. With the aid of a potential function, equipotential $(n+r-1)$-manifolds surrounding $h\left(K^{n}\right)$ in $E^{n+r}$ can be defined [6]. If $h\left(K^{n}\right)$ is twosided in $M^{n+1}$, the intersection $V^{n+r-1} \cap M^{n+1}$ with $M^{n+1}$ of an equipotential sufficiently near $h\left(K^{n}\right)$ falls into two components, $V_{1}^{n}$ and $V_{2}^{n}$, each of which is differentiable and homeomorphic to $K^{n}$. If $h\left(K^{n}\right)$ is one-sided in $M^{n+1}$, points can be so identified in pairs on $V^{n+r-1} \cap M^{n+1}$ as to obtain a differentiable homeomorph of $h\left(K^{n}\right)$.

CoRollary. The $K^{8}$ of Milnor and $K^{10}$ of Kervaire do not admit piecewise differentiable imbeddings in differentiable 9-manifolds and 11-manifolds respectively.

THEOREM 3. If there exists a nonsmoothable $K^{m}$ without boundary, then there is a nonsmoothable $K^{n}$ without boundary for each $n>m$.

In particular, $K^{m} \times S^{1}$ where $S^{1}$ is a circle, is nonsmoothable, for its smoothability would imply that of $K^{m}$, by Theorem 2 . Thus, all the manifolds $K^{8} \times S^{1} \times \cdots \times S^{1}$ and $K^{10} \times S^{1} \times \cdots \times S^{1}$ are nonsmoothable, for Milnor's $K^{8}$ and Kervaire's $K^{10}$.

The invariants used by Milnor and Kervaire are thus freed from the dimensions for which they were defined. They are imbeddability as well as smoothability criteria.

\section{REFERENCES}

1. Morton Brown, $A$ proof of the generalized Schoenflies theorem, Bull. Amer. Math. Soc. vol. 66 (1960) pp. 74-76.

2. M. A. Kervaire, $A$ manifold which does not admit any differentiable structure, Comment. Math. Helv., vol. 34 (1960) pp. 257-270.

3. J. Milnor, On the relationship between differentiable manifolds and combinatorial manifolds, Princeton University, Mimeographed, 1956.

4. Marston Morse, A reduction of the Schoenflies extension problem, Bull. Amer. Math. Soc. vol. 66 (1960) pp. 113-115.

5. Hiroshi Noguchi, The smoothing of combinatorial n-manifolds in $(n+1)$-space. Ann. of Math. vol. 72 (1960) pp. 201-215.

6. René Thom, Les structures differentiables des boules et des sphères, Colloque de Géométrie Différentielle Globale, Centre Belge de Recherches Mathématiques, Brussels, 1959.

UNIVERSITY OF ILLINOIS 